\title{
Comparative evaluation of tubal patency by hysterosalpingography and laparoscopic chromopertubation
}

\author{
Bindu S.* \\ Department of Obstetrics and Gynecology, Government Dharmapuri Medical College, Dharmapuri, Tamil Nadu, India \\ Received: 25 August 2020 \\ Accepted: 10 September 2020 \\ *Correspondence: \\ Dr. Bindu S., \\ E-mail: arvindr84@gmail.com \\ Copyright: (C) the author(s), publisher and licensee Medip Academy. This is an open-access article distributed under \\ the terms of the Creative Commons Attribution Non-Commercial License, which permits unrestricted non-commercial \\ use, distribution, and reproduction in any medium, provided the original work is properly cited.
}

\begin{abstract}
Background: Infertility is defined as one year of unprotected intercourse without pregnancy, primary in which no previous pregnancies have occurred and secondary in which a prior pregnancy not necessarily a live birth has occurred. The objective of the study was to compare the relative efficacy of hysterosalpingography (HSG) and laparoscopy with chromopertubation in the diagnosis of tubal factors in infertile women.

Methods: 90 infertile women attending the infertility clinic at Dharmapuri Medical College and Hospital were selected for this study. The study period was from April 2016 to July 2017 these patients were initially counseled along with their partners and a thorough history of both the partners was obtained followed by a general and pelvic examination of female partners.

Results: HSG results indicated that 48 patients had tubal pathology and 11 patients had uterine pathology. Of those 11 patients with uterine pathology, 6 patients with synechiae had both tubal and uterine pathology. The remaining 5 had only uterine pathology and tubes were patent in them. The site of tubal occlusion in all those blocked tubes shows that more number of the tubal blockage was seen in mid segment of the tube ( 24 cases) followed by the fimbrial block in 16 cases. Diagnostic laparoscopy with chromopertubation was performed in all 90 patients and the findings were recorded. Conclusions: HSG has reasonably good sensitivity and specificity in diagnosing tubal pathology of infertile women. But given the high rate of false-positive diagnosis of tubal pathology (29\%) in HSG, a follow-up laparoscopy is warranted.
\end{abstract}

Keywords: Hysterosalpingography, Luteal phase defect, Salpingitis isthmica nodosa, Pelvic inflammatory disease

\section{INTRODUCTION}

Being labelled infertile is devastating to a couple. For the vast majority of patients, infertility is an unexpected blow to their future. They become desperate for help to achieve their goal of pregnancy and a child. ${ }^{1}$ Many times the problem of infertility has been blamed as the main reason for marital breakdown. Intermittent uterine contractions occur during reproductive years. ${ }^{2}$ These appear to be stimulated by estrogen and modified by progesterone. Uterine contractions may be involved in the rapid transport of sperm through the reproductive tract and play a role in implantation. ${ }^{3}$ The function of the uterus as an incubator for the growing fetus is well known. ${ }^{4}$ The process of implantation requires adequate stimulation of the endometrium first by estrogen, then by the combined activity of estrogen and progesterone. Both endometrium and underlying myometrium must be anatomically and functionally normal to accommodate blastocyst attachment and nidation. ${ }^{5}$ With the development of newer therapies for infertility the chances for treating various infertility problems and the prospects of having a live child is improving. ${ }^{6}$ But with the addition of new investigating modalities in the infertility armamentarium, the cost of investigating the infertile couple has proportionately increased. Many of the treatment failures are due to the failure of compliance due to the final constraints of the couple. 
The purpose of this study is to critically evaluate the usefulness of the 2 important diagnostic modalities namely hysterosalpingography (HSG) and laparoscopy in the diagnosis of infertility as a means to diagnose and treat this malady to a successful conclusion. ${ }^{7}$

\section{METHODS}

90 infertile women attending the infertility clinic at Dharmapuri Medical College and Hospital were selected for this study. The study period was from April 2016 to July 2017 these patients were initially counseled along with their partners and a thorough history of both the partners was obtained followed by a general and pelvic examination of the female partner. Basic investigations like haemoglobin $(\mathrm{Hb} \%)$, urine analysis, blood venereal disease research laboratory (VDRL) test and blood sugar were done. A Mantoux test was performed in female partners whenever necessary.

Patients were carefully selected after excluding the contraindications for HSG and by laparoscopy.

\section{Inclusion criteria}

The study included patients with primary infertility, age between 20 and 40 years, duration of infertility at least 1 year in younger age group, not suffering from other medical illness, and normal seminal and other parameters in the partner.

\section{Exclusion criteria}

The study excluded patients with age more than 40 years and less than 20 years, duration of infertility less than 1 year in younger women, secondary infertility active pelvic inflammatory disease (PID), active cervical or vaginal infection and other medical and surgical disorders.

After the initial evaluation of the patient and her partner, HSG was performed between the 9th and 11th days of the cycle. It was done as an operative (OP) procedure. No severe complications were met with barring few cases of low abdominal pain in the study. Laparoscopy was done in the secretory phase of the menstrual cycle patient was admitted a day before the procedure. All preoperative preparations were done. Laparoscopy was done under general anesthesia. A thorough inspection of the uterus, tubes, ovaries, and cul-de-sac was done.

Findings noted chromopertubation was done by injecting the dye and the nature of the spill visualized. The site of block, hydrosalpinx, and other pathology was noted. At the end of the procedure, an endometrial biopsy was taken for dating and to rule out tuberculosis. The laparoscope was then withdrawn after the completion of the procedure and carbon dioxide let out. The patient was transferred to the post-operative ward after checking pulse, blood pressure (BP), and respiration. She was allowed oral feeds after 6 hours and was discharged after 24 hours.

\section{Statistical analysis}

The data were entered in Epi data and analyzed by IBM Statistical Package for the Social Sciences (SPSS) version 21 software. The data has been presented using descriptive statistics for the prevalence of menopause-related symptoms, health-seeking behavior, and other variables. Bivariate analyses have been done to examine the relationship between predictor and outcome variables.

\section{RESULTS}

All our patients were categorized according to the age distribution which shows that nearly $83 \%$ of the patients were within 30 years age group. Patients who sought medical advice after 35 years were very minimum of 1 which accounts for $1 \%$ of the patients (Table 1 ).

Table 1: Age distribution of infertility.

\begin{tabular}{|lll|}
\hline Age (in years) & Number & Percentage \\
\hline $\mathbf{2 0 - 2 5}$ & 53 & 58.9 \\
\hline $\mathbf{2 5 - 3 0}$ & 25 & 27.8 \\
\hline $\mathbf{3 0 - 3 5}$ & 11 & 12.2 \\
\hline $\mathbf{3 5}$ & 1 & 1.1 \\
\hline
\end{tabular}

The analysis of duration of infertility shows that 3/4th of patients (75.6) sought medical advice within 5 years of marriage. Patients with duration of infertility more than 5 years contributed to only $22 \%$. Only 2 patients in our study group had a duration of infertility for more than 10 years (Table 2).

Table 2: Duration of infertility.

\begin{tabular}{|lll|}
\hline Years & Total numbers & Percentage \\
\hline $\mathbf{1 - 1 5}$ & 68 & 75.6 \\
\hline $\mathbf{6 - 1 0}$ & 20 & 22.2 \\
\hline $\mathbf{> 1 0}$ & 2 & 2.2. \\
\hline
\end{tabular}

Thus with HSG it was found that 48 patients had tubal pathology, 11 patients had uterine pathology. Of those 11 patients with uterine pathology, 6 patients with synechiae had both tubal and uterine pathology. The remaining 5 had only uterine pathology and tubes were patent in them (Table 3).

Table 3: Pathologic causes of infertility as diagnosed by HSG.

\begin{tabular}{|l|l|}
\hline Pathology & Number \\
\hline Tubal pathology & 48 \\
\hline Uterine pathology & 11 \\
\hline Both tubal and uterine pathology & 6 \\
\hline
\end{tabular}

The site of tubal occlusion in all those blocked tubes shows that more number of the tubal blockage was seen in mid segment of the tube ( 24 cases) followed by the fimbrial block in 16 cases. Diagnostic laparoscopy with 
chromopertubation was performed in all 90 patients and the findings were recorded (Table 4).

Table 4: Site of tubal block in HSG.

\begin{tabular}{|ll|}
\hline Site of tubal block & Number \\
\hline Proximal tubal block & 8 \\
\hline Block at mid segment & 24 \\
\hline Fimbrial block & 16 \\
\hline
\end{tabular}

The complete evaluation of tubal, peritoneal, and uterine factors by laparoscopy focused upon the various factors responsible for infertility. Thus the tubal factors were suspected in 34 cases. Uterine pathology contributed to 7 cases. The possible role of anovulation as confirmed by histopathological examination report in 26 cases. Endometriosis accounted for 6 cases where laparoscopy was instrumental (Table 5).

Table 5: Causes of infertility.

\begin{tabular}{|lc|}
\hline Cause of infertility & Number \\
\hline Tubal factors & 34 \\
\hline Uterine factors & 7 \\
\hline Ovarian factors & 26 \\
\hline Endometriosis & 6 \\
\hline
\end{tabular}

The evaluation of tubal factors after laparoscopic chromopertubation shows that the tubes were patent in 56 patients while tubal blockage was noted in 34 patients as compared to 48 and 42 in HSG (Table 6).

Table 6: Patency of tubes as diagnosed by laparoscopic chromopertubation.

\begin{tabular}{|lll|}
\hline $\begin{array}{l}\text { Laparoscopic } \\
\text { chromopertubation }\end{array}$ & Number & Percentage \\
\hline Patent & 56 & 62.2 \\
\hline Blocked & 34 & 37.8 \\
\hline
\end{tabular}

When comparing the tubal patency in HSG and laparoscopic chromopertubaion, it can be seen that more than half of the tubes were found to be patent in laparoscopic chromopertubation $(62.2 \%)$ whereas it was only $47 \%$ on HSG. Blocked tubes constituted $37.8 \%$ in laparoscopy and when compared to HSG the numbers were less (Table 7).

Table 7: Comparison of tubal patency by HSG and laparoscopic chromopertubation.

\begin{tabular}{|c|c|c|c|c|}
\hline \multirow{2}{*}{$\begin{array}{l}\text { Tubal } \\
\text { patency }\end{array}$} & \multicolumn{2}{|c|}{ HSG } & \multicolumn{2}{|c|}{$\begin{array}{l}\text { Laparoscopic } \\
\text { chromopertubation }\end{array}$} \\
\hline & $\mathbf{N}$ & $\%$ & $\mathbf{N}$ & $\%$ \\
\hline $\begin{array}{l}\text { Patent } \\
\text { tube }\end{array}$ & 42 & 47 & 56 & 62.2 \\
\hline $\begin{array}{l}\text { Blocked } \\
\text { tube }\end{array}$ & 48 & 53 & 34 & 37.8 \\
\hline
\end{tabular}

The site of the tubal blockage is mainly the mid-segment as revealed by both diagnostic methods. Laparoscopy could demonstrate only half of those fimbrial blocks which were diagnosed by HSG (Table 8 ).

Table 8: Comparison between HSG and laparoscopic chromopertubation in the site of tubal blockade.

\begin{tabular}{|llll|}
\hline $\begin{array}{l}\text { Site of tubal } \\
\text { blockade }\end{array}$ & Proximal & $\begin{array}{l}\text { Mid- } \\
\text { segment }\end{array}$ & $\begin{array}{l}\text { Fimbrial } \\
\text { end }\end{array}$ \\
\hline HSG & 8 & 24 & 16 \\
\hline Laparoscopy & 5 & 20 & 9 \\
\hline
\end{tabular}

About the patency of the tube HSG and laparoscopy correlate $75 \%$ while blocked tubes have an agreement of $71 \%$. Hence it can be seen that all those patients with patent tubes in HSG were confirmed by laparoscopic chromopertubation. More number of tubes were found to be patent in laparoscopy when compared to HSG. This increase in the number of patent tubes may be due to the release of tubal spasm by anesthesia during laparoscopic chromopertubaion (Table 9).

\section{Table 9: Correlation of HSG findings with laparoscopic chromopertubation.}

\begin{tabular}{|llll|}
\hline & HSG & \multicolumn{3}{l|}{ Laparoscopic chromopertubation } \\
\hline $\begin{array}{l}\text { Patent } \\
\text { tube }\end{array}$ & 42 & 42 & Disagreement \\
\hline $\begin{array}{l}\text { Blocke } \\
\text {-d tube }\end{array}$ & 48 & 34 & 14 \\
\hline
\end{tabular}

Additional pathology diagnosed during laparoscopy in those cases with patent tubes are hydrosalpinx-1, tubo ovarian mass-2, peritubal adhesion-5, total-8.

The sensitivity of HSG as a screening test is - $81 \%$, specificity- $71 \%$, percentage of false positive- $29 \%$. Percentage of false negative-19\%.The initial evaluation of tubal pathology in infertile women by HSG shown a sensitivity of $80.9 \%$ and a specificity of $70.8 \%$. A false positive rate of $28.16 \%$ is seen which implies that whenever a tube is found to be blocked, a laparoscopic chromopertubation has to be done to diagnose or refute the pathology (Table 10).

Table 10: Comparative analysis of tubal pathology between HSG and laparoscopy.

\begin{tabular}{|c|c|c|}
\hline \multirow[t]{2}{*}{ HSG } & \multicolumn{2}{|c|}{$\begin{array}{l}\text { Tubal pathology as diagnosed by } \\
\text { laparoscopy }\end{array}$} \\
\hline & Tubal pathology & Normal tubes \\
\hline Blocked tubes & 34 & 14 \\
\hline Patent tubes & 8 & 34 \\
\hline
\end{tabular}

\section{DISCUSSION}

Abnormalities of the uterus are a relatively uncommon cause of infertility but should always be considered. If for 
no other reason, they can adversely affect the outcome of pregnancies achieved by the successful treatment of more common male, ovarian, and tubal factors. ${ }^{8}$ In Cundiff's study, the pregnancy rate below the age of 31 was $74 \%$. This decreased to $62 \%$ at age 31 to $35 \%$ and $54 \%$ when older than 35. An American study demonstrated a similar relationship with age. The probability of having a healthy baby decreased by $3.5 \%$ per year after age 30 . A woman age 35 had a $50 \%$ chance of having a healthy baby compared to a woman of 25 years. ${ }^{9}$ Similar to the distribution of infertility, the duration of infertility also has a bearing in the management. The majority of the patients in our study sought medical advice within 5 years of practicing unprotected coitus. Only 2 patients found to have a duration of infertility for $>12$ years. Infertility can place psychological stress on the couple to the extent that psychological intervention may become necessary. Infertility is defined as the inability to conceive during 1 year of unprotected intercourse. ${ }^{10}$ In Hurd et al reviewed the conception time for 5574 fertile women $14.6 \%$ of fertile women did not become pregnant during 1 year of unprotected intercourse. All of these women in this study ultimately became pregnant but $6.6 \%$ of the fertile woman required more than 2 years to do so. Various studies have reported the incidence of tubal factors as a cause of female infertility in about $30-40 \%$ of cases. ${ }^{11}$ HSG has the additional advantage of detecting anomalies of the uterine cavity whereas laparoscopy provides an overall picture of the pelvic structure ovary pathology along with tubal patency and architecture. The findings in HSG in our study showed 48 patients to have blocked tubes out of 90 patients. Uterine pathology was seen in 11 patients accounting for $12.2 \%$ of cases of infertility. ${ }^{12}$ Among 6 patients with blocked tubes showed co-existing pathology suggestive of intrauterine synechiae. HSG results were classified as normal, abnormal (bilateral distal tubal obstruction) or suspicious (all others).HSG was confirmed surgically in $96.6 \%$ of normals, $63.1 \%$ of suspicious, and $95.7 \%$ of abnormal. Associated moderate - the severe pelvic disease was found in $16.2 \%$ of normal, 53.9 of suspicious, and $81.7 \%$ of abnormal. ${ }^{13}$ Abnormal HSGs are highly predictive of severe pelvic disease and do not require laparoscopy. Patients with suspicious HSG frequently have normal tubes but poor predictive value for the tubal or associated pelvic disease so requiring confirmatory laparoscopy. Normal HSG has a high negative predictive value which is high enough to warrant diagnostic laparoscopy. ${ }^{14}$ Either a water-soluble contrast medium or oil-based contrast medium has an advantage .water soluble contrast medium is more rapidly absorbed and no risk of lipid embolism or lipid granuloma formation oil-based dyes are associated with less uterine cramping, better resolution of tubal architecture and a higher postprocedure pregnancy rate. HSG diagnosed bilateral proximal in 15 and bilateral distal in 13 patients and mixed in five cases. Diagnostic laparoscopy confirmed the above sites of the block in nine, seventy-one, and three cases. ${ }^{15}$ In comparison, HSG demonstrated $70 \%$ specificity for accurately diagnosing proximal tubal occlusion. The site of the block has an important bearing especially when surgical correction is planned for the patient. Though in vitro fertilization (IVF) - embryo transfer has provided a therapeutic alternative for those with bilaterally blocked tubes, reconstructive tubal surgery still has a role particularly in those settings where the most modern infertility treatments are not available. ${ }^{16}$ The live birth rates were reported to be high in cases of tubo-tubal anastomosis when compared to fimbrioplasty. Moreover, the incidence of ectopic pregnancy is also increased in cases of surgeries done to correct distal tubal occlusion. Patton and colleagues in a series of microsurgical fimbrioplasties in 40 patients reported total intrauterine pregnancy and ectopic pregnancy rates of $63 \%$ and $8 \%$ respectively after 24 months follow up. ${ }^{17}$ The results of microsurgical tubotubal anastomosis have shown an intrauterine pregnancy rate of $63 \%$ and ectopic pregnancy rates of $3.7 \%$ Phelps et al. Majority of the patients studied in our group had a mid-segmental block (24 patients) followed by 16 patients with fimbrial block. Proximal tubal block accounted for 8 patients only. The use of laparoscopy and chromopertubation will show a better picture in the evaluation of infertility. ${ }^{18}$ Diagnostic laparoscopy and chromopertubation and endometrial biopsy was done in all 90 cases for whom HSG was done. In our study, we can find that tubal factors were seen in $38 \%$ of cases while anovulation as a cause of infertility was seen in 26 cases as suggested by histopathological examination 2 cases of tubo ovarian mass and 1 case of hydrosalpinx were additionally diagnosed in laparoscopy in those with a patent tube in HSG. ${ }^{19}$ Moreover, 22 cases of peritubular adhesion were noted of these 5 had shown patent tubes. Endometriosis was diagnosed in 6 cases by laparoscopy $(6.7 \%)$. 7 cases of cystic ovaries were diagnosed and all these showed anovulation by histopathological examination. The other pathologies seen were subserous fibroid in 5 cases bicornuate uterus in one patient and arcuate uterus in 2 patients. More than one factor was seen in some patients. ${ }^{20}$ Viswanath did a study to find the incidence of pathological factors not revealed by HSG but disclosed by laparoscopy in 50 infertile women. The study shows an agreement in $90 \%$ of cases. In a total of 980 tubes examined, HSG identifies fimbrial conglutination in 79 tubes $(8 \%)$ compared to 154 tubes (15.7\%) by laparoscopy. HSG diagnose $68.8 \%$ of the peritubular adhesion confirm by laparoscopy. Endometriosis in 124 women and isolated periovarian adhesions in 48 women by laparoscopy. ${ }^{21}$ Wang et al assessed the diagnostic benefit of laparoscopy in infertile women with normal HSG in 86 patients. Laparoscopy may be omitted in women with normal HSG since it was not changed the original treatment indicated by HSG in $95 \%$ of patients. However, laparoscopy should be recommended in cases with suspected bilateral occlusion on HSG since it altered the original treatment plan in $30 \%$ of patients from IVF to induction of ovulation with intrauterine insemination (IUI). The evaluation of tubal patency by HSG and laparoscopic chromopertubation showed that only 34 patients out of 90 had blocked tubes as compared to 48 patients who had blocked tubes in HSG. Thus laparoscopic chromopertubation showed $62.2 \%$ of 
the cases to have patent tubes while analyzing the site of blocks, more fimbrial blocks were seen in HSG when compared to laparoscopy (16 versus 9).Therefore laparoscopy and HSG are complementary to one another in diagnosing tubal and peritoneal factors and any infertility workup gives a more definite diagnosis when HSG findings were supplemented with laparoscopy. ${ }^{22}$ Corson had shown an accuracy of $91 \%$ and a false-positive rate of $9 \%$ in their study comparing HSG and laparoscopy in the evaluation of tubal pathology. Uterine cavity and tubal patency were properly visualized during modified HSG. Our study showed the percentage of false positives to be high $(28.16 \%)$. Hence when HSG finding of a patent tube is seen, one can wait for 3 to 6 months with the additional advantage of hoping for an increase in fertility but it is not so in cases of blocked tubes which requires an early laparoscopy to confirm the diagnosis and for follow up action. ${ }^{23}$ Therefore it has been demonstrated from the study that HSG and laparoscopy with chromopertubation should be considered complementary to each other. Each has the merits and demerits of its own. ${ }^{24}$ Although laparoscopy was better than HSG as a predictor of future fertility, it should not be considered as the perfect test in the diagnosis of tubal pathology. For clinical practice, laparoscopy can be delayed after a normal HSG for at least 6 months since the probability that laparoscopy will show tubal occlusion after a normal HSG is very low. ${ }^{25}$

\section{CONCLUSION}

The results of the study showed that in diagnosing a patent tube the correlation between HSG and laparoscopy is $75 \%$ for patent tubes and $73 \%$ for blocked tubes. The sensitivity of HSG in diagnosing tubal pathology was $81 \%$ while specificity is $71 \%$. A high false-positive rate of $29 \%$ was shown in this study. To conclude, HSG has reasonably good sensitivity and specificity in diagnosing tubal pathology of infertile women. But given the high rate of false-positive diagnosis of tubal pathology (29\%) in HSG, a follow-up laparoscopy is warranted.

Funding: No funding sources Conflict of interest: None declared

Ethical approval: The study was approved by the Institutional Ethics Committee

\section{REFERENCES}

1. Alessi B. Accuracy of hysterosalpingography and laparoscopic hydrogenation in the diagnosis of tubal patency. Fertil Steril. 1996;65(3):673-5.

2. Munro MG, Brill AI, Parker AH. Gynaecologic endoscopy. In: Novack's Gynecology. 2002; 711973.

3. Rao AR. Uterine and cervical factors. In: Chapter-16, The infertility manual, 2nd Edn. 2004;181-96.

4. Baramki TA. Hysterosalpingography. Fertil Steril. 2005;83(6):1595-606.
5. Bello TO. The pattern of tubal pathology in infertile women hysterosalpingography. Ann Afr Med. 2004;3(2):77-9.

6. Mol BW, Swart P, Bossuyt PM, van der Veen F. Is hysterosalpingography an important tool in predicting fertility outcome. Fertil Steril, 1997;67(4):663-9.

7. Dessole S, Meloni GB, Capobianco G, Manzoni MA, Ambrosini G, Canalis GC. A second HSG reduces the use of the selective technique for the treatment of proximal tubal obstruction. Fertil Steril. 2000;73(5):1037-9.

8. Fayez JA, Mutie G, Schneider PJ. The diagnostic value of hysterosalpingography and laparoscopy in infertility investigations. Int J Fertil. 1988;33(2):98101.

9. Cundiff G, Carr BR, Marshburn PB. Infertile couples with a normal hysterosalpingogram. J Reproductive Med. 1995;40(1):19-23.

10. Heeley DL, Trounson AO, Andersen AN. Female infertility causes \& treatment- A review article. The Lancet. 1994;34:1539-44.

11. Hurd WW, Wyckoff ET, Reynolds DB. Patient rotation and resolution of unilateral corneal obstruction during hysterosalpingography. Obstet Gynecol. 2003;101(6):1275- 8.

12. Rice JP, London SN, Olive DL. Re-evaluation of hysterosalpingography in infertility investigation. Obst Gyne. 1986;6(5):719-21.

13. Nakade KD, Deokar SS. Comparative study of laparoscopy and hysterosalpingography in infertility. J Obst Gynec. 1993;2:785-7.

14. Opsat MS, Miller B, Klein TA. The predictive value of hysterosalpingography for tubal and peritoneal infertility factors. Fertile Steril. 1993;60(3):444-8.

15. Bhatla N. Infections as they infect individual organs. Jeffcoat's principles of gynecology. 200;355-74.

16. Howkins, Bourne PI. Churchill Livingstone Publications; 2002;146-52.

17. Swart P, Mol BW, van der Veen F, van Beurden M, Redekop WK, Bossuyt PM. The accuracy of hysterosalpingography in the diagnosis of tubal pathology. Fertile Steril. 1995;64(3):486-91.

18. Phelps JY, Vlahos NP, Zacur HA. Diagnosis and contemporary management of infertility. PostGraduate Clin Obst Gynecol. 1998;18:1-7.

19. Shalev J, Krissi H, Blankstein J, Meizner I, Rafael ZB, Dicker D. Modified HSG during infertility. Fertil Steril. 2000;74(2):372-5.

20. Speroff L, Glass RH, Kase NG. Female infertility. Chapter- 27. Clinical gynecological endocrinology, and infertility, 7th Edn. Baltimore: William and Walkins; 2014: 1013-1068.

21. Karande VC, Pratt DE, Rabin DS, Gleicher N. The limited value of hysterosalpingography in assessing the tubal status and fertility potential. Fertil Steril. 1995;63(6):1167-71.

22. Wang CW, Lee CL, Lai YM, Tsai CC, Chang MY, Soong YK. Comparison of hysterosalpingography and hysteroscopy in female infertility. J Am Assoc Gynecol Laparosc. 1996;3(4):581-4. 
23. Corson SL, Cheng A, Gutmann JN. Laparoscopy in the normal infertile patient: A question revisited. J Am Assoc Gynecol Laparoscopy. 2000;317-24.

24. Glastein IZ, Sleeper LA, Lavy Y, Simon A, Adoni A, Palti Z, Hurwitz A, Laufer N. Observer variability in diagnosis and management of hysterosalpingography. 1997;67(2):233-7.
25. Sharma R, Sharma V. The infertile woman: a study of 120 cases. J Indian Med Assoc. 1991;89(2);31-2.

Cite this article as: Bindu S. Comparative evaluation of tubal patency by hysterosalpingography and laparoscopic chromopertubation. Int J Reprod Contracept Obstet Gynecol 2020;9:3994-9. 